\title{
Evaluation of concurrent chemoradiotherapy for locally advanced NSCLC according to EGFR mutation status
}

\author{
MIKIKO ISHIHARA $^{1}$, SATOSHI IGAWA ${ }^{1}$, JIICHIRO SASAKI ${ }^{2}$, SAKIKO OTANI $^{1}$, TOMOYA FUKUI ${ }^{1}$, \\ SHINICHIRO RYUGE ${ }^{1}$, KEN KATONO $^{1}$, YASUHIRO HIYOSHI ${ }^{1}$, MASASHI KASAJIMA ${ }^{1}$, \\ HISASHI MITSUFUJI ${ }^{1}$, MASARU KUBOTA ${ }^{1}$, MASANORI YOKOBA ${ }^{3}$, MASATO KATAGIRI ${ }^{3}$, \\ AKANE SEKIGUCHI ${ }^{4}$, ITARU SODA ${ }^{4}$, HIROMICHI ISHIYAMA ${ }^{4}$, \\ KAZUSHIGE HAYAKAWA ${ }^{4}$ and NORIYUKI MASUDA ${ }^{1}$

\begin{abstract}
${ }^{1}$ Department of Respiratory Medicine; ${ }^{2}$ Research and Development Center for New Medical Frontiers, Kitasato University School of Medicine, Sagamihara, Kanagawa 252-0374; ${ }^{3}$ School of Allied Health Sciences, Kitasato University, Sagamihara, Kanagawa 252-0373; ${ }^{4}$ Department of Radiology and Radiation Oncology, Kitasato University School of Medicine, Sagamihara, Kanagawa 252-0374, Japan
\end{abstract}

Received March 17, 2016; Accepted January 31, 2017

DOI: $10.3892 / \mathrm{ol} .2017 .6231$

\begin{abstract}
Concurrent chemoradiotherapy (cCRT) is the standard treatment for patients with locally advanced non-small cell lung cancer (LA-NSCLC). However, the efficacy and safety of this treatment has not been compared between patients who possess epidermal growth factor receptor (EGFR) mutations and patients with wild-type EGFR. The objective of the present study was to evaluate the effect of the presence of EGFR gene mutations in patients with LA-NSCLC receiving cCRT. Between January 2007 and December 2013, the records of 64 patients were reviewed retrospectively. The data were statistically analyzed to evaluate the efficacy of cCRT according to EGFR mutation status. In total, 15/64 were revealed to possess EGFR mutations, 23\%, and comprised the mutant EGFR group. The progression-free survival time was significantly shorter in the mutant EGFR group compared with the patient group with tumors exhibiting wild-type EGFR, 6.3 and 9.5 months, respectively $(\mathrm{P}<0.001)$. The overall survival rate was longer in the mutant EGFR group compared with the wild-type EGFR group, although the difference was not statistically significant, 37.1 and 21.1 months, respectively $(\mathrm{P}=0.26)$. The disease recurred in all of the patients of the mutant EGFR group, whilst the recurrence rate in the wild-type EGFR group was $89 \%$. The frequency of distant metastasis was significantly higher in the mutant EGFR group compared with the wild-type EGFR group. In conclusion, these data suggest that additional studies are required to identify
\end{abstract}

Correspondence to: Dr Satoshi Igawa, Department of Respiratory Medicine, Kitasato University School of Medicine, 1-15-1 Kitasato, Minami-ku, Sagamihara, Kanagawa 252-0374, Japan

E-mail: igawa@kitasato-u.ac.jp

Key words: epidermal growth factor receptor mutation, locally advanced non-small cell lung cancer, chemoradiotherapy strategies for reinforcing the efficacy of cCRT, with a focus on the potential use of EGFR tyrosine kinase inhibitors for patients exhibiting an EGFR mutation.

\section{Introduction}

Lung cancer is a leading cause of cancer-associated mortality worldwide (1). Non-small cell lung cancer (NSCLC) accounts for $80 \%$ of all cases of lung cancer, and $30 \%$ of patients with NSCLC present with locally advanced lung cancer. The standard treatment for patients with locally advanced NSCLC (LA-NSCLC) possessing a good Eastern cooperative oncology group performance status (ECOG-PS) (0 or 1$)$ and adequate organ function is thoracic radiotherapy (TRT) combined with chemotherapy $(2,3)$. Previous randomized trials demonstrated that concurrent cytotoxic chemoradiotherapy (cCRT) with a third-generation regimen, a combination of a platinum compound with novel agents, is more effective compared with second-generation regimens $(4,5)$. However, the majority of treated individuals developed disease recurrence, with a 5-year survival rate of $15-20 \%(3,5)$. The identification of somatic gene mutations in the tyrosine kinase domain of the epidermal growth factor receptor (EGFR) $(6,7)$ led to the development of a novel treatment strategy for patients with advanced NSCLC (8-11). Molecular profiling has become essential for the treatment of patients with advanced NSCLC to predict the response to specific molecular targeted agents such as EGFR tyrosine kinase inhibitors (TKIs). Despite the developments in this area of molecular biology, and the discovery of EGFR mutation, there have been no additional improvement in the treatment of LA-NSCLC in the previous decade. In this context, additional research is required to understand the biological behavior of the population of patients with LA-NSCLC with EGFR mutations. The objective of the present study was to evaluate and validate the frequency of EGFR mutations among patients with LA-NSCLC, and the clinical efficacy of cCRT in patients with LA-NSCLC according to EGFR mutation status. 


\section{Patients and methods}

Patient selection. The patients enrolled in the present retrospective cohort study were diagnosed with unresectable LA-NSCLC and received cisplatin-based chemotherapy with cCRT at the Kitasato University Hospital (Sagamihara, Japan) between January 2007 and December 2013. All patients were histologically or cytologically diagnosed with NSCLC, and received a thoracic radiotherapy (TRT) dose of 50-60 Gy. Medical records were reviewed to collect patient data and data associated with the tumors including the age, gender, tumor EGFR mutation status, clinical disease stage, ECOG-PS, smoking history and presence or absence of a history of EGFR-TKI therapy in each patient. The patients were classified according to their smoking status as non-smokers, $<100$ cigarettes in a lifetime, or current/former smokers. All patients with unknown EGFR mutation status and patients who underwent induction chemoradiotherapy followed by definitive surgery were excluded. The present study was carried out with approval from the Institutional Review Board at Kitasato University School of Medicine (Sagamihara, Japan).

Response analysis. Tumor response was classified according to the Response Evaluation Criteria in Solid Tumors (version 1.1) (12). Patients were evaluated to identify the evaluable lesions prior to chemoradiotherapy. If chest radiography suggested disease progression or recurrence, additional detailed examination was performed by computed tomography $(\mathrm{CT})$ scans of the chest and abdomen, and other imaging techniques such as magnetic resonance imaging of the head and fludeoxyglucose positron emission tomography.

Analysis for detecting EGFR mutations. Cytologic or histologic specimens were examined for the presence or absence of EGFR mutations by the peptide nucleic acid (PNA)-locked nucleic acid (LNA) polymerase chain reaction (PCR) clamp method or Cycleave method as previously described $(13,14)$.

Treatment methods. All patients were treated with TRT and 2 cycles of cCRT. TRT was administered in 2 Gy daily standard fractionation using 6 or $10 \mathrm{MV}$ X-rays, depending on the position and size of the individual tumors. The total target dose of radiation was fixed at $60 \mathrm{~Gy}$. A CT-based treatment-planning system was mandatory to define the planning target volume. Dose distribution was calculated with tissue heterogeneity correction. The radiation field was reduced around the primary tumor and involved the lymph nodes, subsequent to exposure to 40 Gy using adequate fields to limit the dose to the spinal cord, which received a maximum dose $\leq 50 \mathrm{~Gy}$. The planned percentages of lung volume receiving $>20 \mathrm{~Gy}$ (V20) was $<35 \%$. cCRT consisted of $80 \mathrm{mg} / \mathrm{m}^{2}$ cisplatin on day 1 and $20 \mathrm{mg} / \mathrm{m}^{2}$ vinorelbine on days 1 and 8 . Subsequently, the patients received 2 cycles of consolidation chemotherapy with $80 \mathrm{mg} / \mathrm{m}^{2}$ cisplatin on day 1 and $25 \mathrm{mg} / \mathrm{m}^{2}$ vinorelbine on days 1 and 8 .

Statistical analysis. The differences in the response rates and recurrence patterns according to the tumor EGFR mutation status were compared using the $\chi^{2}$ test. Progression-free survival (PFS) was measured from the date of start of cCRT to the date of documentation of treatment failure; death, disease progression or appearance of unacceptable toxicity, or the date of censoring at the last follow-up examination. Overall survival (OS) was defined as the interval between the date of start of cCRT and date of death from any cause or the date of censoring. Post-progression survival (PPS) was measured from the date of documentation of disease progression to the date of death from any cause or the date of censoring. The survival curves were plotted using the Kaplan-Meier method, and the differences between the survival times were analyzed using the log-rank test. The variables, including gender, smoking status, PS, status of EGFR mutation, histology and clinical stage were used as variables in a Cox's proportional hazards model to determine the hazard ratios for OS and PPS. $\mathrm{P}<0.05$ was considered to indicate a statistically significant difference, and statistical analysis was performed using SPSS, version 17.0 (SPSS, Inc., Chicago, IL, USA) for Windows.

\section{Results}

Patient characteristics. The data of 64 patients with unresectable LA-NSCLC who received cCRT with cisplatin-based chemotherapy were examined. The median follow-up time was 27.4 months. The main clinical characteristics of the patients are summarized in Table I. Amongst the 64 patients, 15 (23\%) patients possessed EGFR mutations in the tumor. EGFR mutations in the tumors were observed predominantly in non-smokers. All the patients possessed an ECOG-PS of 0-1, and the distribution of the clinical stage was not statistically different between the mutant and wild-type EGFR groups.

Response and survival. The objective tumor responses are summarized in Table II. The overall response rate was $73.4 \%$. The response rates (RRs) in the patient groups with mutant and wild-type EGFR in the tumors were 66.7 and $75.5 \%$, respectively, and no statistically significant difference was observed. The median time to achieve the objective response was not significantly different between the mutant and wild-type EGFR groups, 1.25 and 1.28 months, respectively. The crude recurrence rates in the mutant and wild-type EGFR groups were $100(15 / 15)$ and $89 \%$ (44/49), respectively. The frequency of distant metastasis was significantly higher $(\mathrm{P}=0.01)$ in the mutant EGFR group compared with the wild-type EGFR group (Table III).

The survival data are demonstrated in Fig. 1. A significantly shorter PFS was observed in the mutant EGFR group compared with the wild-type EGFR group, median PFS 6.3 and 9.5 months, respectively $(\mathrm{P}<0.001)$. Conversely, there was no significant difference in OS observed between the two groups, although OS tended to be longer in the mutant EGFR group, median OS 37.1 and 21.1 months, respectively $(\mathrm{P}=0.26)$. The PPS data are demonstrated in Fig. 2. Amongst the patients who exhibited disease relapse, the PPS was significantly longer in the mutant EGFR group compared with the wild-type EGFR group, median PPS 29.9 and 11.2 months, respectively $(\mathrm{P}=0.015)$.

The results of multivariate analysis are summarized in Table IV. The presence of EGFR mutation and diagnosis of clinical stage were independent prognostic factors of short PFS. Additionally, the presence of EGFR mutation tended to be a predictor of a long PPS. 
Table I. Patient characteristics.

\begin{tabular}{|c|c|c|c|}
\hline \multirow[b]{2}{*}{ Characteristics } & \multicolumn{2}{|c|}{ EGFR mutation (\%) } & \\
\hline & Mutant (n=15) & Wild type $(n=49)$ & \\
\hline \multicolumn{4}{|l|}{ Age } \\
\hline Median, range & $61,52-72$ & $60,34-74$ & \\
\hline \multicolumn{4}{|l|}{ Gender } \\
\hline Male & $10(67)$ & $38(78)$ & \\
\hline Female & $5(33)$ & $11(22)$ & \\
\hline \multicolumn{4}{|l|}{ Smoking status } \\
\hline Non-smoker & $8(53)$ & $6(12)$ & \\
\hline Current/former smoker & $7(47)$ & $43(88)$ & $\mathrm{P}<0.001$ \\
\hline \multicolumn{4}{|l|}{ ECOG-PS } \\
\hline $0-1$ & $15(100)$ & $49(100)$ & \\
\hline $2-4$ & 0 & 0 & \\
\hline \multicolumn{4}{|l|}{ Clinical stage } \\
\hline IIIA & $7(47)$ & $22(45)$ & \\
\hline IIIB & $8(53)$ & $27(55)$ & \\
\hline \multicolumn{4}{|l|}{ Chemotherapy regimen } \\
\hline Platinum based regimen & $15(100)$ & $49(100)$ & \\
\hline Presence of EGFR-TKI therapy & $15(100)$ & $3(0.12)$ & $\mathrm{P}<0.0001$ \\
\hline
\end{tabular}

WHO, World Health Organization; ECOG, Eastern Cooperative Oncology Group performance status; EGFR, epidermal growth factor receptor; TKI, tyrosine kinase inhibitor.

Table II. Tumor response.

\begin{tabular}{|c|c|c|c|c|}
\hline \multirow[b]{2}{*}{ Variables } & \multirow[b]{2}{*}{ All patients $(n=64)$} & \multicolumn{2}{|c|}{ EGFR mutation } & \multirow[b]{2}{*}{ P-value ${ }^{a}$} \\
\hline & & Mutant (n=15) & Wild type $(n=49)$ & \\
\hline Complete response & 2 & 0 & 2 & \\
\hline Partial response & 45 & 10 & 35 & \\
\hline Stable disease & 12 & 5 & 7 & \\
\hline Progressive disease & 4 & 0 & 4 & \\
\hline Not evaluable & 1 & 0 & 1 & \\
\hline Response rate $(\%)$ & 73.4 & 66.7 & 75.5 & 0.84 \\
\hline
\end{tabular}

${ }^{\mathrm{a}} \chi^{2}$ test; Mutant vs. Wild type; EGFR, epidermal growth factor receptor. Response rate $=$ complete response + partial response.

Table III. Recurrence rate and recurrence pattern.

\begin{tabular}{lccc}
\hline & \multicolumn{2}{c}{ EGFR mutation } & \\
\cline { 2 - 3 } Variables & $\begin{array}{c}\text { Mutant } \\
(\mathrm{n}=15)\end{array}$ & $\begin{array}{c}\text { Wild type } \\
(\mathrm{n}=49)\end{array}$ & P-value \\
\hline Crude recurrence rate (\%) & $15(100)$ & $44(89)$ & \\
Loco-regional recurrence & 1 & 15 & 0.06 \\
Distant recurrence & 14 & 29 & 0.01 \\
\hline
\end{tabular}

EGFR, epidermal growth factor receptor.

\section{Discussion}

The present study evaluated the effect of EGFR mutation in patients with LA-NSCLC who underwent cCRT with definitive radiotherapy. In the present study, PFS was revealed to be significantly shorter in the EGFR mutant group compared with the wild type group. Disease recurrence was revealed to occur more frequently in the EGFR mutant group compared with the wild type group, particularly more frequently in the distant site from the primary region.

Other studies have reported on the association between EGFR status and treatment outcomes subsequent to cCRT in 

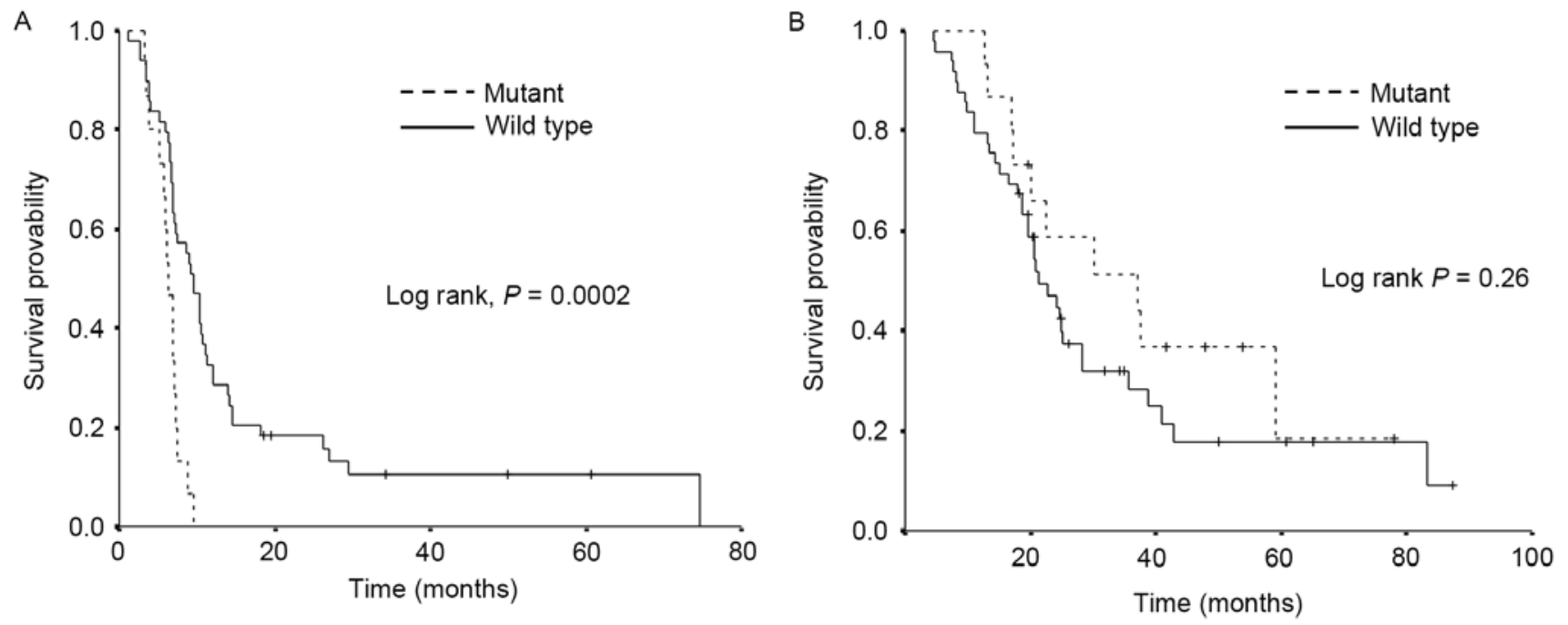

Figure 1. Kaplan-Meier plots of the (A) progression-free survival and (B) overall survival in patients with (EGFR) mutations in the tumor vs. patients with wild-type EGFR in the tumor. EGFR, epidermal growth factor receptor; bold line, EGFR mutation group; dashed line, EGFR wild type.

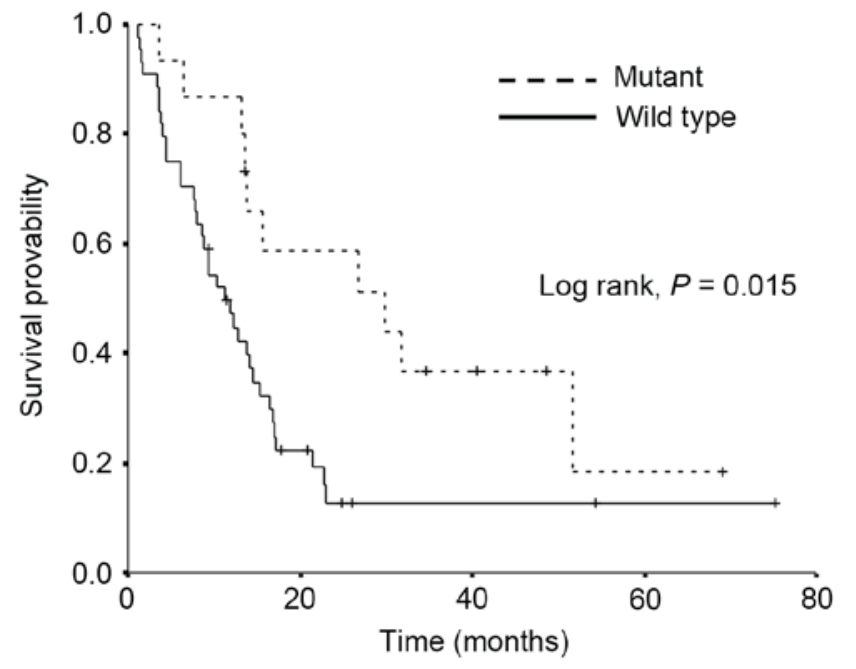

Figure 2. Kaplan-Meier plots of the post-progression survival in patients with EGFR mutations in the tumor vs. patients with wild-type EGFR in the tumor. EGFR, epidermal growth factor receptor; bold line, EGFR mutation group; dashed line, EGFR wild type.

patients with LA-NSCLC. Amongst these studies, the majority of groups reported that PFS and recurrence rate were not statistically different (15-17) between the two groups. In the present study PFS was shorter and recurrence rate was higher in the EGFR mutant group, which was inconsistent with data from previous studies. Only one study, Tanaka et al (18), demonstrated that the PFS was shorter and 2-year recurrence-free survival rate was poorer in the EGFR mutant group.

Notably, the recurrence patterns in these studies subsequent to cCRT in LA-NSCLC setting were similar to those of the present study, which demonstrated that the EGFR mutant groups exhibited lower loco-regional recurrence rate $(15,16,18)$. Preclinical studies have demonstrated that NSCLC cell lines with EGFR mutations exhibit greater radiosensitivity compared with those without EGFR mutation. The assumed mechanism underlying this observation is the delayed repair of radiation-induced DNA damage in these cells $(19,20)$. These preclinical studies may measure the aforementioned clinical observations, better local control of the cCRT for EGFR mutant patients, as the anticancer effect for irradiated field is superior for the EGFR mutant tumors.

Although these observations promote the expectation that cCRT is a potentially more favorable treatment in patients with LA-NSCLC that possess EGFR mutations, these studies do not demonstrate that CCRT is more beneficial compared with PFS. Additionally, the PFS was significantly shorter in the EGFR mutant group in the present study. To explain this discrepancy, the recurrence rate in the distant site was a focus of the present study. It was significantly higher in the EGFR mutant group compared with the wild type $(\mathrm{P}=0.01)$. Therefore, it may be hypothesized that the EGFR mutant tumors were more likely to result in distant metastasis. These data regarding recurrence patterns are considered important as it suggests that the improvement of the treatment strategy for patients with EGFR mutant LA-NSCLC is required.

Regarding OS, the present study demonstrated a tendency towards a longer median OS and a significantly longer median PPS in the mutant EGFR group compared with the wild-type EGFR group. Previous studies have revealed that in patients with metastatic NSCLC who possess EGFR mutations, EGFR-TKI therapy was associated with higher response rates and a longer PFS compared with standard chemotherapy (8-11). Therefore, the present study hypothesized that the addition of EGFR-TKI therapy alongside standard chemotherapy may account for the longer OS and PPS in the mutant EGFR group compared with the wild-type EGFR group in the groups of the present study. The multivariate analysis of PPS performed in the present study supports this hypothesis. Several studies have reported that EGFR inhibition enhances the antitumor activity of ionizing radiation in vitro (21-23). Accordingly, it may be appropriate to suggest that a combined therapy of EGFR inhibitor and TRT may improve the probability of a cure in patients with LA-NSCLC who possess an EGFR mutation. However, few clinical trials have been able to demonstrate a clear benefit of combined EGFR-TKI therapy with TRT in patients with 
Table IV. Multivariate analysis using a Cox's proportional hazards regression model.

\begin{tabular}{llr}
\hline A, PFS & \multicolumn{2}{c}{ Multivariate analysis } \\
\hline & \multicolumn{1}{c}{ HR $(95 \%$ CI $)$} & P-value \\
Variables & $1.07(0.50-2.25)$ & 0.87 \\
\hline Gender & $0.95(0.51-1.77)$ & 0.87 \\
ECOG-PS (0 vs. 1) & $0.79(0.35-1.76)$ & 0.56 \\
Smoking (current/former smoker vs. non-smoker) & $3.23(1.51-6.88)$ & $<0.01$ \\
EGFR (mutant vs. wild type) & $1.94(1.07-3.52)$ & 0.03 \\
Clinical stage (IIIA vs. IIIB) & $1.06(0.48-2.30)$ & 0.89 \\
Histology (Sq. vs. Non-Sq.) & & \\
\hline
\end{tabular}

\section{B, PPS}

\begin{tabular}{llr}
\hline & \multicolumn{2}{c}{ Multivariate analysis } \\
\cline { 2 - 3 } Variables & HR (95\% CI) & P-value \\
\hline Gender & $1.45(0.64-3.26)$ & 0.38 \\
ECOG-PS (0 vs. 1) & $1.15(0.55-2.40)$ & 0.72 \\
Smoking (current/former smoker vs. non-smoker) & $0.93(0.35-2.46)$ & 0.89 \\
EGFR (mutant vs. wild type) & $0.41(0.16-1.03)$ & 0.06 \\
Clinical stage (IIIA vs. IIIB) & $0.79(0.43-1.45)$ & 0.44 \\
Histology (Sq. vs. Non-Sq.) & $1.17(0.50-2.78)$ & 0.71 \\
\hline
\end{tabular}

PFS, progression-free survival; PPS, post-progression survival; ECOG-PS, Eastern Cooperative Oncology Group performance status; EGFR, epidermal growth factor receptor; Sq, squamous cell carcinoma; HR, hazard ratio; CI, confidence interval.

LA-NSCLC (24-29). In an attempt to obtain an answer to this question, the WJOG6911L trial is currently in progress in Japan, which is a multicenter phase II trial of gefitinib administration in combination with radiotherapy in patients with LA-NSCLC who possess sensitizing EGFR mutations (trial no., UMIN000008366).

The data of the present study demonstrated a higher recurrence rate in the EGFR mutant and wild type groups compared with historical control data of phase III studies of cCRT $(3,4)$. It is hypothesized that two of the eligibility criteria of the patients may have caused this difference. Firstly, the result of the EGFR mutation analysis was required for the present study, which was possibly absent if a patient survived without disease recurrence. Secondly, patients who were treated with induction chemoradiotherapy followed by definitive surgery $(\mathrm{CRT}+\mathrm{S})$ were excluded. In Kitasato University Hospital (Sagamihara, Japan), CRT+S was performed for the patients in which the tumors were nearly resectable stage IIIA tumors that had been successfully downgraded in stage, and had become resectable with a good response for the induction chemoradiotherapy. It is suggested that exclusion of these patient groups who exhibited better prognoses caused the higher recurrence rate.

There were several limitations to the present study. First, as a retrospective study the results cannot be regarded as definitive. Secondly, the sample size may not have been sufficient. Thirdly, there was no pharmacokinetic validation for the differences in the efficacy of chemotherapy according to the tumor EGFR mutation status in the present study. In addition, the eligibility criteria of the patients included a result of the EGFR mutation status, which may have introduced some bias during patient selection. The absence of EGFR mutation analysis was most commonly due to the histological diagnosis of the patients, such as squamous cell carcinoma.

In conclusion, the present study confirmed that conventional cCRT using platinum based regimen may not be the most effective type of treatment for patients with LA-NSCLC who possess EGFR mutations. The results of ongoing studies including the WJOG6911L trial are required to identify novel strategies for improving the efficacy of cCRT, with special consideration given to the potential use of EGFR-TKIs.

\section{Acknowledgements}

The authors would like to thank the staff members in the Department of Respiratory Medicine, Kitasato University School of Medicine (Sagamihara, Japan) for their suggestion and assistance. The present study was supported by Grant-in-Aid for Scientific Research (C) (grant no. 26461896) from the Japan Society for the Promotion of Science (Tokyo, Japan). 


\section{References}

1. Siegel R, DeSantis C, Virgo K, Stein K, Mariotto A, Smith T, Cooper D, Gansler T, Lerro C, Fedewa S, et al: Cancer treatment and survivorship statistics, 2012. CA Cancer J Clin 62: 220-241, 2012.

2. Pritchard RS and Anthony SP: Chemotherapy plus radiotherapy compared with radiotherapy alone in the treatment of locally advanced, unresectable, non-small-cell lung cancer. A meta-analysis. Ann Intern Med 125: 723-729, 1996.

3. Furuse K, Fukuoka M, Kawahara M, Nishikawa H, Takada Y, Kudoh S, Katagami N and Ariyoshi Y: Phase III study of concurrent versus sequential thoracic radiotherapy in combination with mitomycin, vindesine, and cisplatin in unresectable stage III non-small-cell lung cancer. J Clin Oncol 17: 2692-2699, 1999.

4. Segawa Y, Kiura K, Takigawa N, Kamei H, Harita S, Hiraki S, Watanabe Y, Sugimoto K, Shibayama T, Yonei T, et al: Phase III trial comparing docetaxel and cisplatin combination chemotherapy with mitomycin, vindesine, and cisplatin combination chemotherapy with concurrent thoracic radiotherapy in locally advanced non-small-cell lung cancer: OLCSG 0007. J Clin Oncol 28: 3299-3306, 2010

5. Yamamoto N, Nakagawa K, Nishimura Y, Tsujino K, Satouchi M, Kudo S, Hida T, Kawahara M, Takeda K, Katakami N, et al: Phase III study comparing second- and third-generation regimens with concurrent thoracic radiotherapy in patients with unresectable stage III non-small-cell lung cancer: West Japan Thoracic Oncology Group WJTOG0105. J Clin Oncol 28: 3739-3745, 2010

6. Lynch TJ, Bell DW, Sordella R, Gurubhagavatula S, Okimoto RA, Brannigan BW, Harris PL, Haserlat SM, Supko JG, Haluska FG, et al: Activating mutations in the epidermal growth factor receptor underlying responsiveness of non-small-cell lung cancer to gefitinib. N Engl J Med 350: 2129-2139, 2004.

7. Paez JG, Jänne PA, Lee JC, Tracy S, Greulich H, Gabriel S, Herman P, Kaye FJ, Lindeman N, Boggon TJ, et al: EGFR mutations in lung cancer: Correlation with clinical response to gefitinib therapy. Science 304: 1497-1500, 2004.

8. Maemondo M, Inoue A, Kobayashi K, Sugawara S, Oizumi S, Isobe $\mathrm{H}$, Gemma A, Harada M, Yoshizawa $\mathrm{H}$, Kinoshita I, et al: Gefitinib or chemotherapy for non-small-cell lung cancer with mutated EGFR. N Engl J Med 362: 2380-2388, 2010.

9. Mitsudomi T, Morita S, Yatabe Y, Negoro S, Okamoto I, Tsurutani J, Seto T, Satouchi M, Tada H, Hirashima T, et al: Gefitinib versus cisplatin plus docetaxel in patients with non-small-cell lung cancer harbouring mutations of the epidermal growth factor receptor (WJTOG3405): An open label, randomised phase 3 trial. Lancet Oncol 11: 121-128, 2010.

10. Zhou C, Wu YL, Chen G, Feng J, Liu XQ, Wang C, Zhang S, Wang J, Zhou S, Ren S, et al: Erlotinib versus chemotherapy as first-line treatment for patients with advanced EGFR mutation-positive non-small-cell lung cancer (OPTIMAL, CTONG-0802): A multicentre, open-label, randomised, phase 3 study. Lancet Oncol 12: 735-742, 2011.

11. Rosell R, Carcereny E, Gervais R, Vergnenegre A, Massuti B, Felip E, Palmero R, Garcia-Gomez R, Pallares C, Sanchez JM, et al: Erlotinib versus standard chemotherapy as first-line treatment for European patients with advanced EGFR mutation-positive non-small-cell lung cancer (EURTAC): A multicentre, open-label, randomised phase 3 trial. Lancet Oncol 13: 239-246, 2012.

12. Eisenhauer EA, Therasse P, Bogaerts J, Schwartz LH, Sargent D, Ford R, Dancey J, Arbuck S, Gwyther S, Mooney M, et al: New response evaluation criteria in solid tumours: Revised RECIST guideline (version 1.1). Eur J Cancer 45: 228-247, 2009.

13. Nagai Y, Miyazawa H, Huqun, Tanaka T, Udagawa K, Kato M, Fukuyama S, Yokote A, Kobayashi K, Kanazawa M and Hagiwara $\mathrm{K}$ : Genetic heterogeneity of the epidermal growth factor receptor in non-small cell lung cancer cell lines revealed by a rapid and sensitive detection system, the peptide nucleic acid-locked nucleic acid PCR clamp. Cancer Res 65: 7276-7282, 2005.

14. Yatabe Y, Hida T, Horio Y, Kosaka T, Takahashi T and Mitsudomi T: A rapid, sensitive assay to detect EGFR mutation in small biopsy specimens from lung cancer. J Mol Diagn 8: 335-341, 2006

15. Akamatsu H, Kaira K, Murakami H, Serizawa M, Koh Y, Ono A, Shukuya T, Tsuya A, Nakamura Y, Kenmotsu H, et al: The impact of clinical outcomes according to EGFR mutation status in patients with locally advanced lung adenocarcinoma who recieved concurrent chemoradiotherapy. Am J Clin Oncol 37: 144-147, 2014
16. Yagishita S, Horinouchi H, Katsui Taniyama T, Nakamichi S, Kitazono S, Mizugaki H, Kanda S, Fujiwara Y, Nokihara H, Yamamoto $\mathrm{N}$, et al: Epidermal growth factor receptor mutation is associated with longer local control after definitive chemoradiotherapy in patients with stage III nonsquamous non-small-cell lung cancer. Int J Radiat Oncol Biol Phys 91: 140-148, 2015.

17. Hayashi H, Okamoto I, Kimura H, Sakai K, Nishimura Y, Nishio K and Nakagawa K: Clinical outcomes of thoracic radiotherapy for locally advanced NSCLC with EGFR mutations or EML4-ALK rearrangement. Anticancer Res 32: 4533-4537, 2012.

18. Tanaka K, Hida T, Oya Y, Oguri T, Yoshida T, Shimizu J, Horio Y, Hata A, Kaji R, Fujita II, et al: EGFR mutationimpact on definitiveconcurrent chemoradiation therapy for inoperable stage III adenocarcinoma. J Thorac Oncol: Sep 2, 2015 (Epub ahead of print).

19. Das AK, Sato M, Story MD, Peyton M, Graves R, Redpath S, Girard L, Gazdar AF, Shay JW, Minna JD and Nirodi CS: Non-small-cell lung cancers with kinase domain mutations in the epidermal growth factor receptor are sensitive to ionizing radiation. Cancer Res 66: 9601-9608, 2006.

20. Das AK, Chen BP, Story MD, Sato M, Minna JD, Chen DJ and Nirodi CS: Somatic mutations in the tyrosine kinase domain of epidermal growth factor receptor (EGFR) abrogate EGFR-mediated radioprotection in non-small cell lung carcinoma. Cancer Res 67: 5267-5274, 2007.

21. She Y, Lee F, Chen J, Haimovitz-Friedman A, Miller VA, Rusch VR, Kris MG and Sirotnak FM: The epidermal growth factor receptor tyrosine kinase inhibitor ZD1839 selectively potentiates radiation response of human tumors in nude mice, with a marked improvement in therapeutic index. Clin Cancer Res 9: 3773-3778, 2003.

22. Chinnaiyan P, Huang S, Vallabhaneni G, Armstrong E, Varambally S, Tomlins SA, Chinnaiyan AM and Harari PM: Mechanisms of enhanced radiation response following epidermal growth factor receptor signaling inhibition by erlotinib (Tarceva). Cancer Res 65: 3328-3335, 2005.

23. Raben D, Helfrich B, Chan DC, Ciardiello F, Zhao L, Franklin W, Barón AE, Zeng C, Johnson TK and Bunn PA Jr: The effects of cetuximab alone and in combination with radiation and/or chemotherapy in lung cancer. Clin Cancer Res 11: 795-805, 2005.

24. Koh PK, Faivre-Finn C, Blackhall FH and De Ruysscher D: Targeted agents in non-small cell lung cancer (NSCLC): Clinical developments and rationale for the combination with thoracic radiotherapy. Cancer Treat Rev 38: 626-640, 2012

25. Okamoto I, Takahashi T, Okamoto H, Nakagawa K, Watanabe K, Nakamatsu K, Nishimura Y, Fukuoka M and Yamamoto N: Single-agent gefitinib with concurrent radiotherapy for locally advanced non-small cell lung cancer harboring mutations of the epidermal growth factor receptor. Lung Cancer 72: 199-204, 2011.

26. Center B, Petty WJ, Ayala D, Hinson WH, Lovato J, Capellari J, Oaks T, Miller AA and Blackstock AW: A phase I study of gefitinib with concurrent dose-escalated weekly docetaxel and conformal three-dimensional thoracic radiation followed by consolidative docetaxel and maintenance gefitinib for patients with stage III non-small cell lung cancer. J Thorac Oncol 5: 69-74, 2010.

27. Rothschild S, Bucher SE, Bernier J, Aebersold DM, Zouhair A, Ries G, Lombrieser N, Lippuner T, Lütolf UM, Glanzmann C and Ciernik IF: Gefitinib in combination with irradiation with or without cisplatin in patients with inoperable stage III non-small cell lung cancer: A phase I trial. Int J Radiat Oncol Biol Phys 80: 126-132, 2011.

28. Ready N, Jänne PA, Bogart J, Dipetrillo T, Garst J, Graziano S, Gu L, Wang X, Green MR and Vokes EE; Cancer, Leukemia Group B, Chicago, IL: Chemoradiotherapy and gefitinib in stage III non-small cell lung cancer with epidermal growth factor receptor and KRAS mutation analysis: Cancer and leukemia group B (CALEB) 30106, a CALGB-stratified phase II trial. J Thorac Oncol 5: 1382-1390, 2010.

29. Choong NW, Mauer AM, Haraf DJ, Lester E, Hoffman PC, Kozloff M, Lin S, Dancey JE, Szeto L, Grushko T, et al: Phase I trial of erlotinib-based multimodality therapy for inoperable stage III non-small cell lung cancer. J Thorac Oncol 3: 1003-1011, 2008. 